\title{
PerFORMance: integrating structural feedback into design processes for complex form-active surfaces
}

\author{
T. Al-Haddad \& T. R. Gentry \\ Georgia Institute of Technology, USA
}

\begin{abstract}
This paper outlines a method of using Finite Element Analysis (FEA) software to study the behavior of materials, geometries, and configurations to create an iterative design feedback loop for generating and refining complex configurations of form-active surfaces. The method integrates technical feedback on structural performance - material stresses, deformations and elastic buckling potential-while respecting design intention and promoting constructability, improved structural performance, and syntactic consistency. Instead of the 2-dimensional (planar) technology which drove modernist analysis towards the structural hyper-rationality of the trabeated system, this new process compiles and synthesizes computational speed, mathematic principles, mechanical knowledge, and material logics within a digital 3-dimensional (spatial) analytical environment in order to realize a new paradigm of constructible complex surface structures. The research focuses on the development of structural performance criteria for form-active structures and interoperability techniques/protocols between advanced CAD systems and advanced structural analysis systems in order to create a fluid design + analysis process of generating and engineering complex form-active designs.

Keywords: FEA, structural analysis, structural surface, shape stiffening, buckling, computation.
\end{abstract}

\section{Introduction}

Today the architectural discipline finds itself having mastered the representational production of blobs, folds, cells, webs, cracks, crystals, fractures, plumes, drips, nests, sponges, shells, et cetera. The explosion of 
formal possibilities created by intuitive geometric modeling environments have lead architects to design many freeform complex surface geometries without concern for constructability or structural feasibility. Now architects are left asking themselves the age-old question: how do we build these forms? This question leads us to the next question: how do we analyze these forms structurally in order to predict how complex surface geometry will actually behave in the physical world? Thus far we have usually seen a material-spatial schism at this point in the design process into a fairly normalized stick frame structural system clad with a complex skin.

This schism is caused in large part by our inability to integrate the structural analysis of complex surface forms into a fluid design process based on a design + analysis feedback loop allowing for structural feasibility and improved system performance that is fully representative of geometry and materiality. This is one of the primary problems in realizing these complex forms in the physical world while maintaining conceptual consistency. However, the technology to analyze these forms mathematically is quite mature and is ready for architectural appropriation. Finite Element Analysis (FEA) is an analytic method for calculating the structural behavior of objects, and is the method by which designers can now work iteratively between formal desires and physical behaviors. By utilizing FEA designers can begin to move towards a more holistic design process where architecture and engineering are collapsed into a singular hybrid.

The problem is rooted in an architectural design philosophy that is hylomorphic in nature. Manuel DeLanda describes this hylomorphic paradigm as "a view of matter as an inert receptacle for forms imposed from the outside" [1]. Designers project their formal desires and intuitions onto materials and generate geometries without regard for the physical behaviors produced by those decisions. This is why most formally complex designs must be 'simplified' and 'normalized' within engineering conventions so that we can predict behavior and protect the public health, safety, and welfare. When built, these designs are normally split into a two-part system of structure and surface, often having little rational relationship between the two systems. If designers had the ability to analyze the structural behavior of their formal creations continuously during the design process, would this information impact formal decision-making? We argue here that the feedback derived from the analysis of material properties and geometric behavior using FEA can and should affect formal decisions in order to create a new paradigm in which complex structuralsurfaces are realizable as self-supporting constructions, also potentially capable of carrying significant external loads. Additionally, analytical structural feedback should be used as a design generator, or collaborative partner, for the creation and articulation of geometric and spatial complexity through intricately articulated form-active surfaces.

Although much more automated, stochastic, and discrete, similar conceptual ideas of incorporating structural feedback into the formal creation process can be found in the work of Kristina Shea's Eifform Software. Eifform is a rule-based formal generation package that incorporates structural behavior into a generative 
algorithm to produce iterative, optimized design solutions based on predetermined performance criteria. This approach considers structural behavior as equal to formal manifestation. The algorithm uses shape instantiation and a process of structural optimization based on crystallization called simulated annealing to create designs that produce 'structural efficiency, economy of materials, member uniformity, and even aesthetics' [2]. While not directly related to FEA the parallels between the work presented here and the Eifform strategy are clear.

\section{Finite Element Analysis}

Finite Element Analysis (from here forth referred to as FEA) is a mathematical method for analyzing the behavior of form and matter based on an approximate discretized representation of a desired condition with topologically connected 1D, 2D, and 3D Finite Elements. This method was developed for the aerospace industry in the 1950 s to replace the traditional method of 2D/3D truss analysis and to reduce the need for empirical testing through full-scale mockups. Because the existing method of analysis assumed limited types of behavior it worked well for simple trusses and frames. However, it was not suitable for complex forms because of the unpredictable nature of complex shapes. The advent of digital computing made the difficulty of analyzing complex 3D shapes possible through FEA. The Boeing Corporation was the first to successfully analyze a complex surface with an early version of FEA in the 1950s [3].

Finite Element Modeling is structured into a three-part environment of preprocessor, solver, and post-processor. Pre-processors are used for generating the FE mesh from CAD geometry and setting up the necessary conditions for completing the analysis. Once the model has been completed in the preprocessor, a FE input deck is normally written out to a text file and submitted to the solver where the mathematical abstraction is assembled and the model is solved. The solver then writes a results file that can be viewed in the postprocessing environment [4].

Depending on the nature of the analysis, the results could include the deformations of the structure under loading, the stress state of the materials in the structure, the buckling factors (eigenvalues) and buckled mode shapes (eigenvectors) and vibration frequencies and mode shapes (another form of eigenvalues and eigenvectors). A linear-elastic FEA is most commonly used and cannot assess whether the structure is damaged due to yield/fracture of the materials or whether the structure has undergone wholesale geometric changes due to excessive deformation. A non-linear FEA can model complex behavior such as crushing and post-buckling, but such behaviors are rarely permissible in architectural structures - so such non-linear analyses are rarely used. The most "accessible" forms of structural feedback are the stresses, which indicate whether the materials are performing within safe limits. Deformations are also easy to understand, with designers being able to use intuitive judgment to assess whether the movements of the structure under various loads are acceptable. Buckling and 
vibration are complex structural phenomena - but must be considered if thinskinned complex membrane forms are to be considered.

One of the most important aspects of this process is the fluid translation of information from the design environment (CAD) to the analytical environment (FEA) and back again. This translation is critical to the usefulness of FEA as an iterative design tool. In the past, FE models where built manually in a preprocessor environment or in a text editor. This was a time consuming, tedious, and error prone process. Every node (point where the continuum is broken into discreet parts) had to be manually input through coordinate definition and then elements created through nodal connectivity. One can easily see why complex forms would not be easily analyzed in this type of constructive environment. However, today algorithms have been created to automatically generate FE models from well-formed CAD geometry, opening the door for designers to analyze complex forms quickly and with less specialized knowledge.

The key to making this process successful from a CAD perspective is to have well-formed, clean geometry that does not contain anomalous conditions. In addition to geometric fitness, CAD files should be well organized into layering schema that will aid in the meshing process down stream. In single body models this is less of a concern, but in multi-body models containing connections this can be the difference between hours and days of pre-processor work. In multibody, connected models, all points of connection should be geometrically identified and organized into discrete layers in the CAD model. This will greatly simplify modeling connectors in the pre-processor. Lastly, geometric aides can be modeled in CAD to aid in a variety of pre-processor tasks. For example, lines can be created to establish vectors where loads will be generated in the preprocessor. In general, it is advantageous to create as much geometric and spatial information within the $\mathrm{CAD}$ environment as possible because pre-processors are still quite clumsy in comparison. Once the geometric model is complete it should be exported to a neutral file format that is supported by the pre-processor such as IGES, DXF, or ACIS.

After CAD geometry has been imported into the pre-processor the model should then be organized into component layers, material properties, and Boundary Conditions. Once the basic organizational schema has been built meshing can begin.

Automeshing techniques have been one of the key factors in making FEA a viable design tool. Automeshing is the procedure by which raw surface or solid geometry is translated into a variety of different $2 \mathrm{D}$ shell/plate elements or $3 \mathrm{D}$ solid elements. The resolution of the discretization process is controlled by the designer, but the software calculates the three-dimensional coordinates of the nodes and keeps track of element connectivity. This mesh is analogous to a tessellated surface mesh in a CAD environment. The elements can be of either first order or second order type, each containing up to 20 independent nodes. Second order elements generally give better results but are more computationally expensive. Automeshing maps FE elements to geometry and creates a single collector (layer) that is organized into geometry and elements. The retention of this relationship between the element and its originating geometry is critical for a 
variety of reasons including the ability to refine the mesh of elements after geometric manipulation and for mapping connectors, constraints, and loads.

Automeshing often needs to be refined in order to obtain high quality results for final engineering checks. However, for initial design analysis most automeshing algorithms yield acceptable results. The exercise in Section 4 will only deal with the meshing of 2D shell elements, which are analogous to thin surface structures.

Once meshing is complete, property attributes for elements and materials should be defined. For 2D shell elements the most important property to define is the virtual thickness. The thickness is considered virtual because the thickness is constant within the formulation of the element and does not need to be modeled in CAD. In addition, most solvers require the user to give instructions on how to deal with elements as either shells (2D) or solids (3D). Material properties must then be defined and mapped to the proper elements. Most linear static FE analyses define materials as being homogeneous and isotropic. Other material properties such as Modulus of Elasticity and Poisson's Ratio must also be defined [5]. These material properties are easily accessible in a variety of engineering references for common structural materials.

The final step in setting up the model in the pre-processor is the creation of Boundary Conditions, from here on referred to as BCs. BCs are locations where loads (forces) and fixed points (constraints) are defined within the model. Loads can consist of many different types of forces; points loads, pressures, accelerations, moments, etc, and are all applied directly to individual elements or nodes. The study below will focus on uniform loads of a constant magnitude. This loading schema will be representative of gravity loads. Constraint points describe how the model is fixed in space. The structural solution will provide a force (or moment) reaction at each point fixity. In three-dimensional models, every point (node) has six degrees of freedom (DOF), three translational and three rotational. For every constraint, each of the six DOF can be independently defined. Models which are inadequately constrained are unstable, that is, when the forces are applied to them, they move as a rigid body instead of deforming and remaining in one place.

Once the model has been constrained and a variety of forces have been applied, Load Steps, or Load Combinations, are created. Load Steps allow for multiple loading permutations to be solved for a single structural construct.

The model is now ready to be solved. An FE input deck is written out from the pre-processor and then submitted to the solver for analysis. Once the analysis is complete, the solver will output a results file based on the results that were requested from within the pre-processor. This results file is then read into the post-processor for visualization and results analysis [6].

The results obtained in the post-processor from the results file become the structural feedback data that designers can use to modify and develop geometric conditions based on performance and design intensions. The format of the result is critical for designers to be able to intuitively react to the analysis. Rather than outputting hundreds of pages of nodal displacements as text, contemporary 
post-processors can generate sophisticated plots, graphs, spreadsheets, and animated simulations of shape behavior along with many other forms of userfriendly representation.

\section{Structural criteria for form-active surfaces}

FEA is capable of analyzing many different types of scenarios and behaviors including temperature gradients, acceleration, vibration, etc. In order for the process of design, analysis, and feedback to be productive, set criteria must be established for the architect to react against. These criteria should be intuitively comprehensible and must also be highly generative. In other words, the resultant data must be useful for the architect in making decisions about which formal operations to use where, and why those operations are useful. The two primary feedback criteria to be used in this process are material stresses and buckling. Material stresses and buckling modes will be analyzed using gravity-loaded shell structures to predict behavior and search for design opportunities. These design opportunities are defined as moments identified through FEA where shapestiffening formal manipulations will contribute to the geometric realization and overall structural performance of the project.

Analysis of material stresses are important in order to ensure that the limit state of the material is not exceeded so that rigidity is retained. A safety factor of one-half the material limit state is typically used in designing for stresses. For ductile materials such as steel, the limit state is usually defined by the yield point. FEA calculates stresses in the geometry and displays the results as easily read colored contour plots in order to consider the effects of stresses on materials and in making dimensional decisions.

Buckling is the phenomena where an object with internal compressive stresses gives way and drastically changes its shape. It is caused by an instability in the structure. Buckling phenomena can be explained and predicted by using stress values to formulate an eigenproblem. Eigenproblems yield eigenvalues and eigenvectors. The eigenvalues are the load multipliers that describe the load level at which the structure buckles, along with the original loading pattern. The eigenvectors give the pattern of displacement of the structure as it buckles. The eigenvectors are only a visual pattern of the displacement - the magnitude of this pattern cannot be determined and it is generally considered to be infinite - that is, the onset of buckling is a catastrophic failure of the structure. FEA will produce both colored contour plots and simulated animations in order to visualize buckling behavior along with determining the BLF (Buckling Load Factor) The BLF is the multiplier of the applied load at which the structure will begin to buckle.

Once the FE mesh has been loaded and an analysis has been run the results of the analysis can be read by the designer as feedback for modifications and development. Areas of large deformation would require the most shapestiffening operations and therefore would present design opportunities manifest as shape-stiffening operations. These operations can come in the form of folds, bulges, knots, and many other techniques that increase the amount of $3 \mathrm{D}$ shape 
in the project geometry. This is where design intension and designer's intuition come into play. The objective of this process is not to optimize the shape solely based on structural performance, but rather to create an iterative dialog between designer's intuitions/desires and structural behavior.
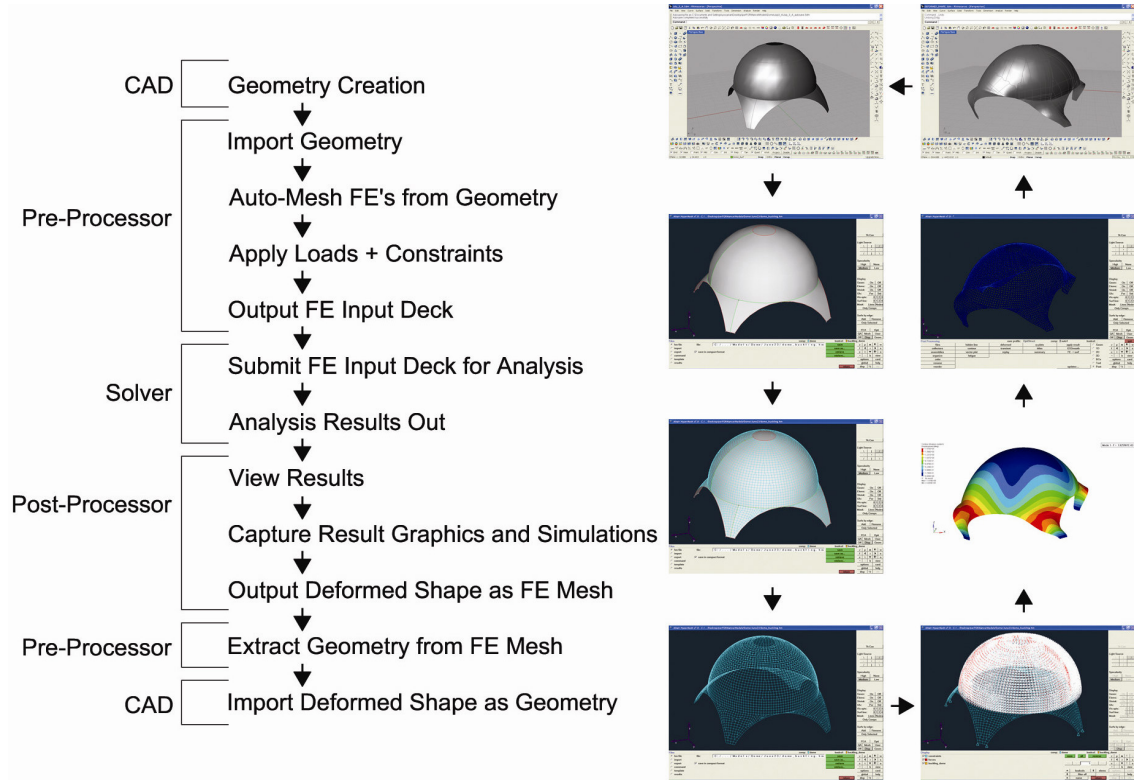

Figure 1: $\quad$ Process of translation from CAD to FEA.
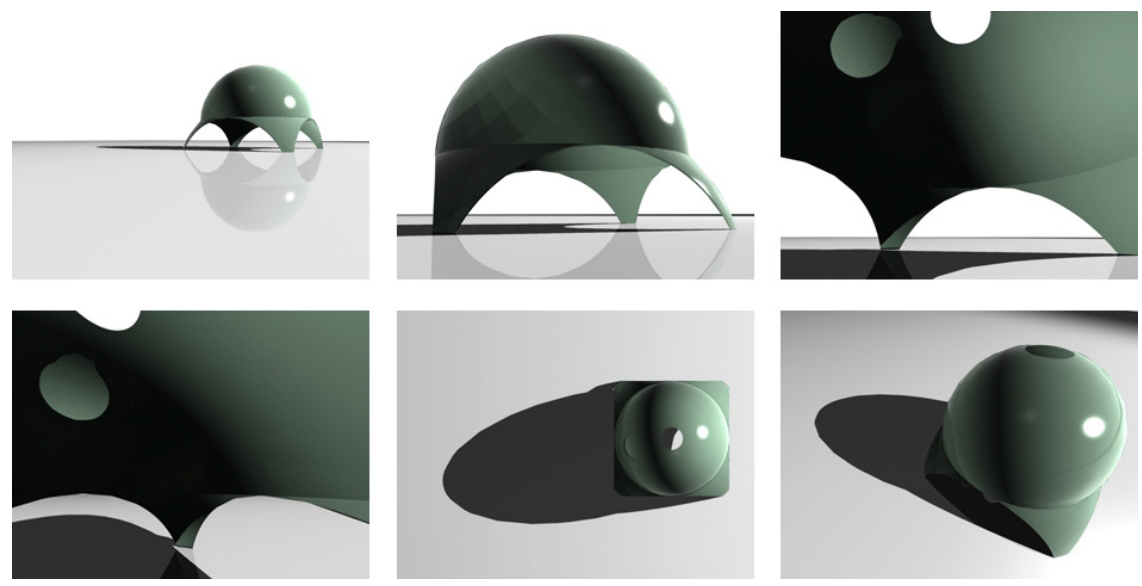

Figure 2: Original dome geometry. 


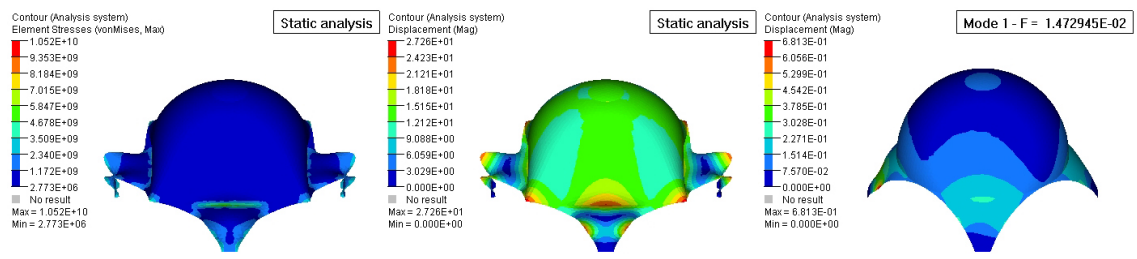

Figure 3: Analytical results for dome.

\section{Design experiment: folding the dome}

The following design experiment tests the premise of using FEA as structural feedback in the iterative design of a self-supporting structural skin. Starting with a semi-spherical dome, the experiment is set up as a prototypical transformation of an archetypical form. The original dome has a span of $43 \mathrm{~m}$, as taken from the dimensions of the Pantheon in Rome, and is set on top of four pendentive arches. The thickness of the surface is $0.00635 \mathrm{~m}(6.35 \mathrm{~mm})$ and is modeled with the properties of mild steel. After the CAD model was converted to a FE mesh, the FE model was loaded with a total uniform load of $5.3 \times 10^{6} \mathrm{~N}$, which is the equivalent to the load that would be produced by taking the area of the structure's footprint and loading it with a generically derived load of $60 \mathrm{lbs} / \mathrm{ft}^{2}$. This loading is arbitrary as the project has neither program nor specific occupancy, and is the combination of a $40-1 \mathrm{~b} / \mathrm{ft}^{2}$ live load and a $20-\mathrm{lb} / \mathrm{ft}^{2}$ dead load.

The base of each pendentive was then continuously fixed at every FE node and given zero DOF. An analysis was then run on the dome for buckling, stress, and displacement. The results of this analysis were captured, considered, and then used in the transformation of the dome.
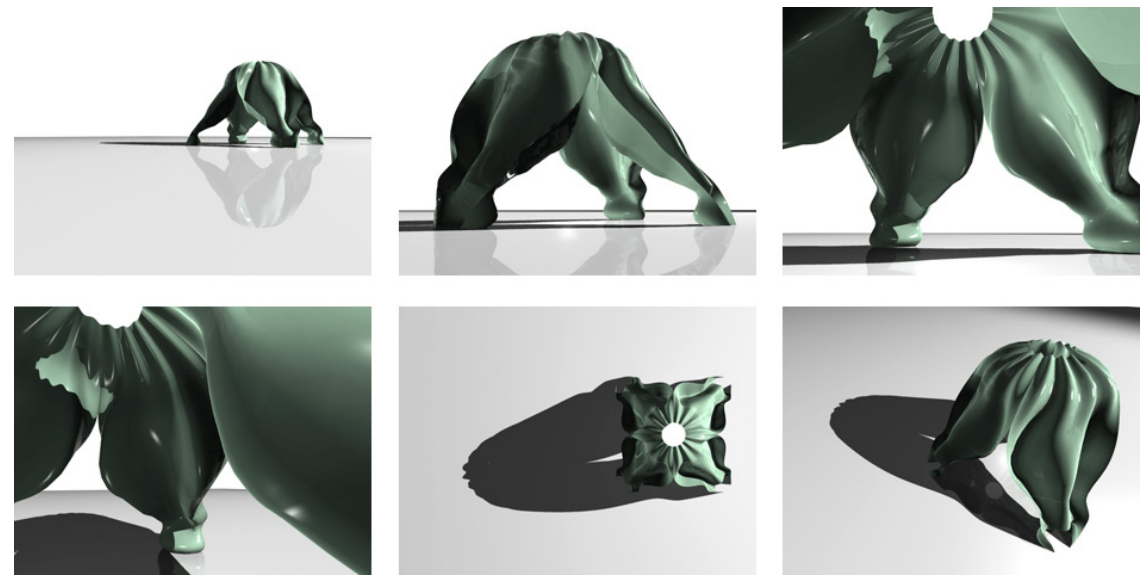

Figure 4: First iteration dome transformation. 

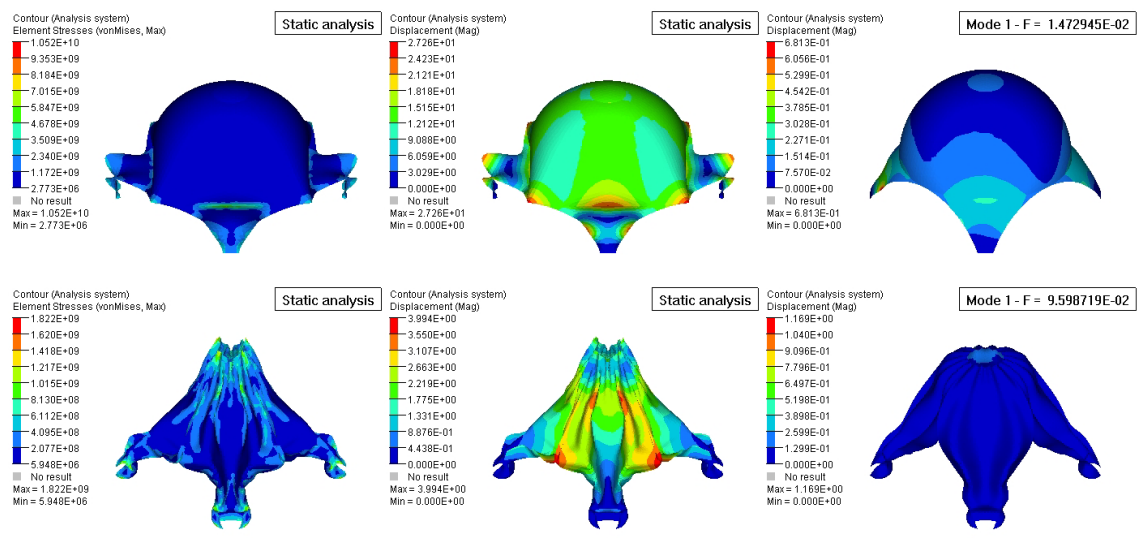

Figure 5: Comparison of analysis between original and first transformation.

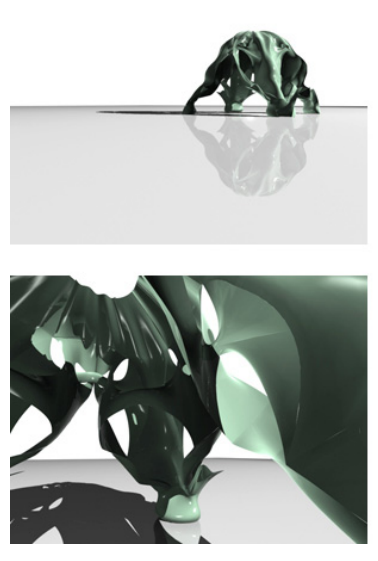

Figure 6: Second iteration dome transformation.

Each transformational iteration tries to introduce shape-stiffening operations in areas of high deformation. Geometric complexity and surface continuity are gradually produced as overall stiffness is increased.

Decision making for which formal operations would be used to modify the initial geometry came from a mixture of designer's intensions/intuitions and analytical results from the FE dome model. Analysis of the original geometry shows buckling behavior in the pendentive legs that identified an opportunity for shape-stiffening operations. Additionally, the upper portion of the dome was shown to be performing quite well with the original geometry. Therefore a strategy of lofting from deep curves in the lower regions to shallow curves in the upper region was implemented. 
The original geometry was first superimposed with the deformed geometries from the buckling analyses to give a spatial frame of reference to the designer. This is of course an exaggerated representation, however it is an intuitive context for spatial thinkers. The shapes were then deconstructed and used to generate deep sinuous curves that were then lofted to produce the four folded legs in the next formal iteration. The curves were drawn perpendicular to the line of intersection produced by slicing a plane through the dome on a $45^{\circ}$ diagonal in plan. In other words, deep curves were drawn perpendicular to the original dome and then swept, using lofts, 1-rail sweeps, 2-rail sweeps, and surface blends. The material thickness remained constant throughout all iterations, more or less maintaining an equivalent usage of material.
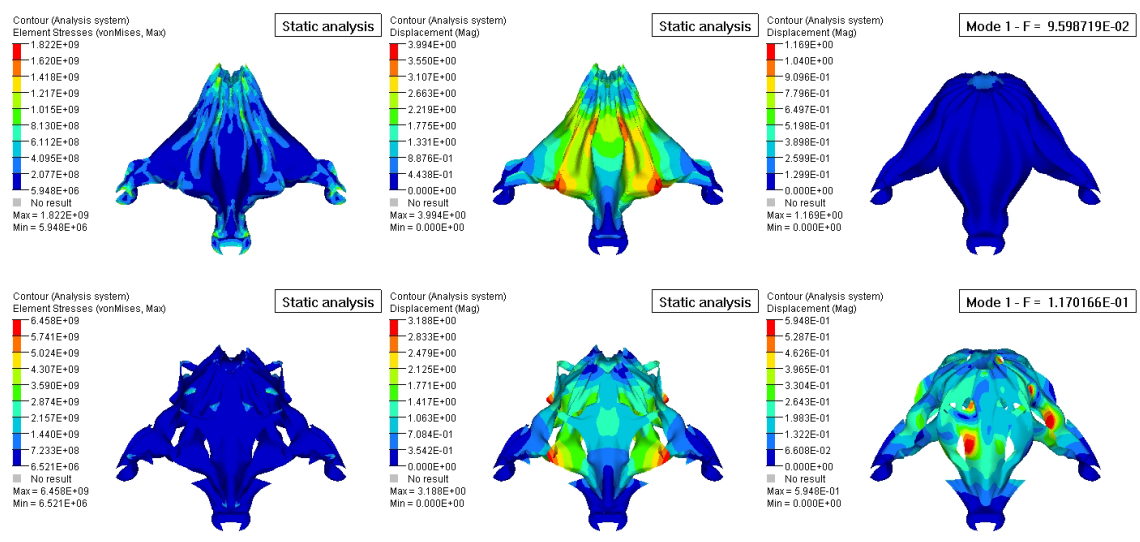

Figure 7: Comparison of analysis between first and second transformation.
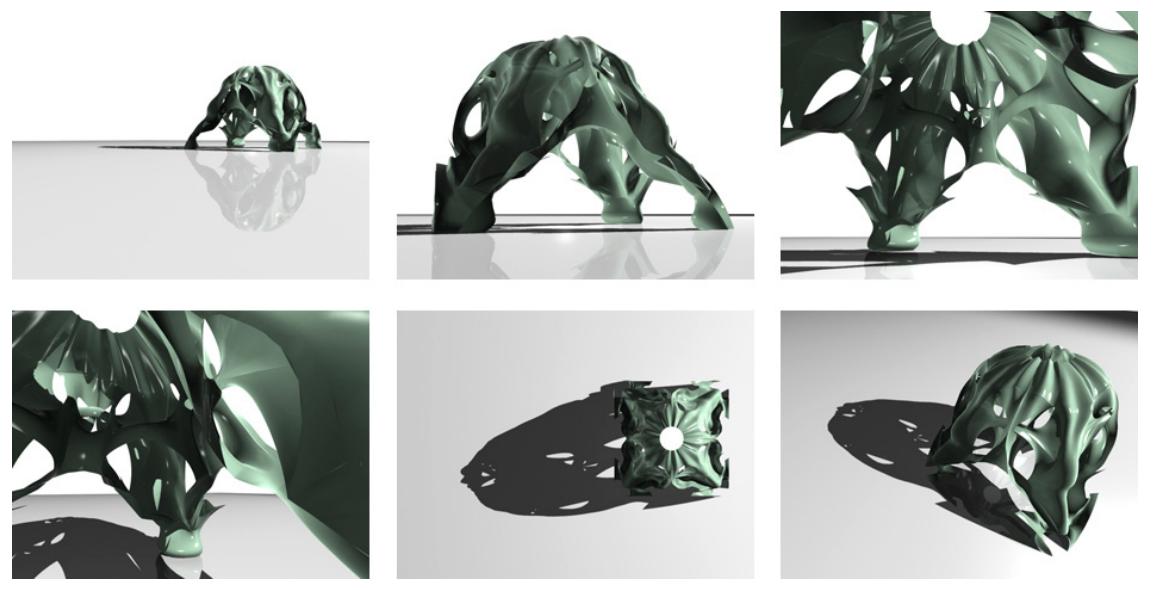

Figure 8: Third iteration dome transformation. 


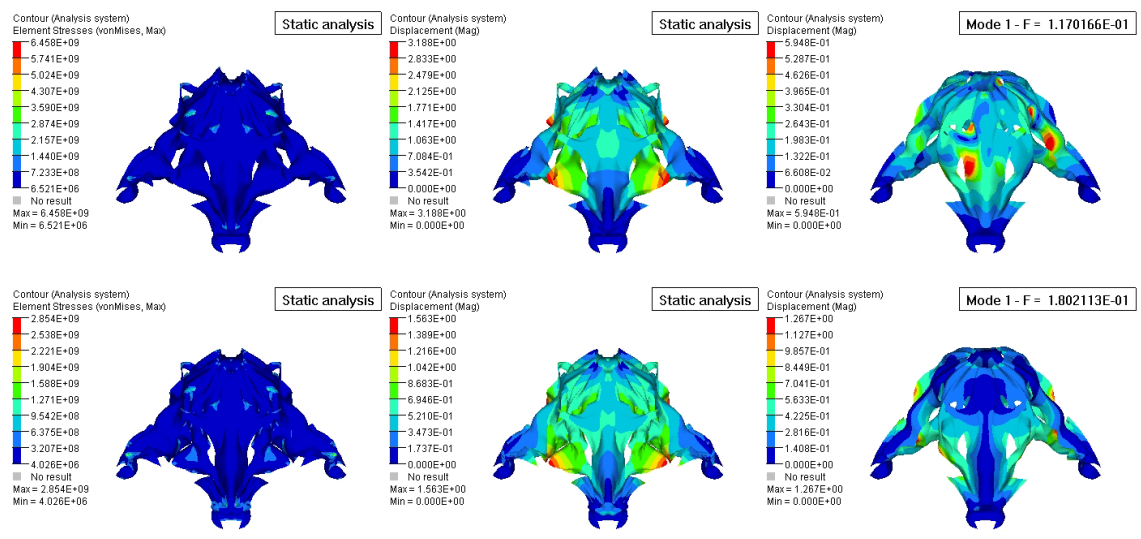

Figure 9: Comparison of analysis between second and third transformation.
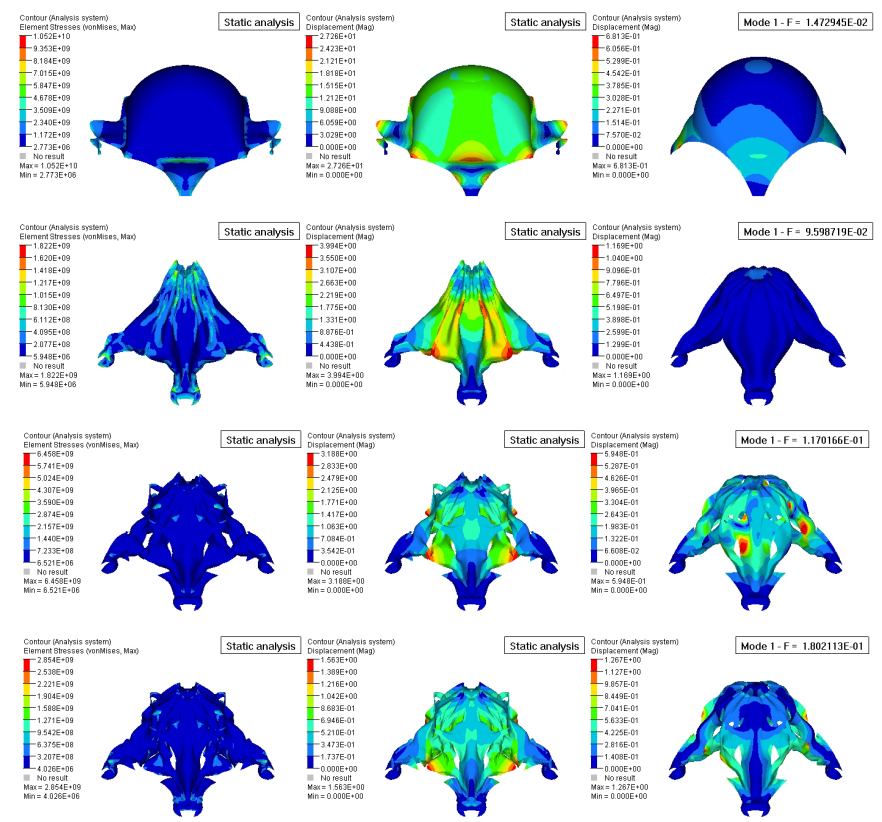

Figure 10: Comparison of analytical results for all shapes.

Analysis of the first transformation showed an improvement in global buckling behavior but also identified potential for local buckling in some surfaces. Local buckling indicates an instability that is restricted to a small region of the structure, as opposed to global buckling that indicates a wholesale (and usually catastrophic) change in geometry. The next iteration tried to 
improve global buckling further by connecting the lower areas of each arch and by introducing more shape in areas prone to local buckling.

Analysis of the second transformation again showed improved stiffness but the form still needed additional stiffening in the middle areas shown above as yellow and red in Figure 7. The final iteration increases surface connectivity globally by making smooth toroidal transitions across previously unconnected surfaces.

Ultimately, comparative analysis between all four geometries above shows that with each set of shape stiffening operations the structural performance increases significantly. The BLF has increased from 0.015 for the dome to 0.18 for the final iteration. The BLF for the second and third iterations is 0.096 and 0.117 respectively. This marks a twelve-fold increase in stiffness from the original geometry to the final geometry. Additionally, maximum displacements have also been significantly reduced from 27.3 units to 1.6 units, marking a 17 fold decrease in maximum displacements.
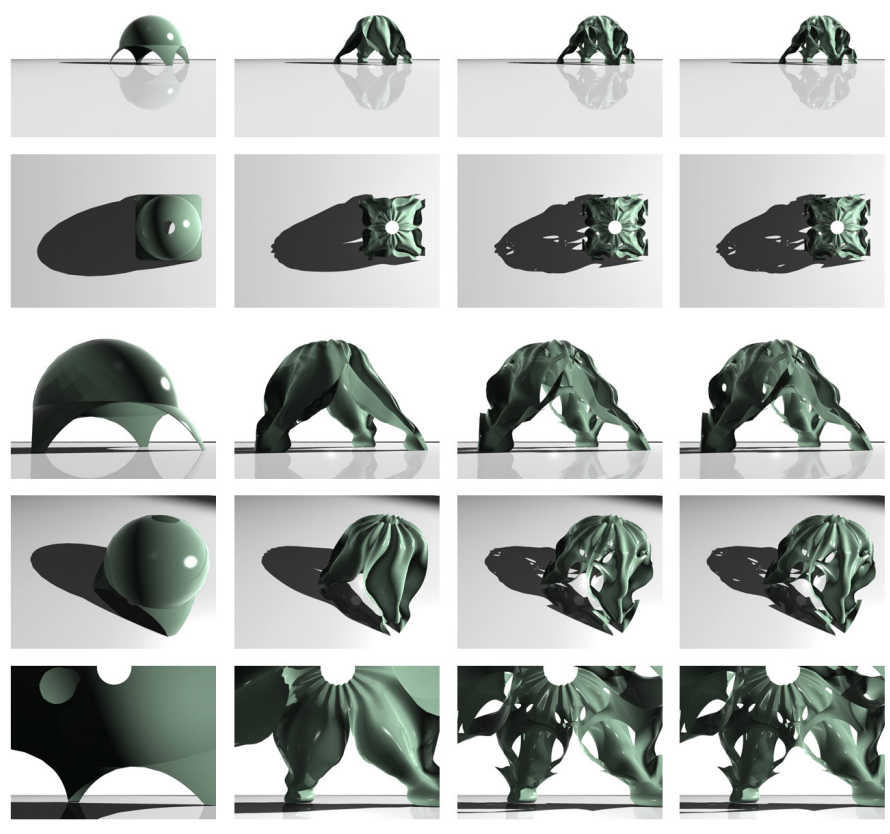

Figure 11: Progression of geometric transformation.

\section{Conclusion}

The process presented here can be taken as an analytical example for designers wanting to use material behavior and shape stiffness as primary drivers for designing and building complex surface geometries. FEA is a clear verification of the premise that $3 \mathrm{D}$ shape increases stiffness. Through this process geometric 
complexity can also be significantly increased. Formal aesthetics, design intensions/intuitions, geometry, material, and physical behavior have all converged in this process to produce a rich form where structure and skin, concept and construct, and process and product can be understood simultaneously through a continuum of surface.

Excluding the time necessary for geometric modeling, each of these FE analyses took approximately $30 \mathrm{~min}$ to setup, solve, and format the results. This is a powerful demonstration of how this method could very feasibly be incorporated into a daily design cycle. One workday could easily produce 3-4 geometric possibilities, depending on geometric complexity, with relatively accurate behavioral models demonstrating structural feasibility and potentials for further geometric development. Additionally, as true parametric modeling is quickly becoming normalized in design offices, one could easily imagine producing 10-20 geometric and analytical daily derivations for a project.

The deployment of this technology and these techniques as presented above is immediate. FEA is over 50 years old now and has already found its way into the background of many CAD packages that are used on desktop machines in architectural offices. Only desire is needed to implement this technique. The potential for future research lies in using FEA to design with non-linear, dynamic behaviors in mind. This method shows tremendous promise for developing even richer structures that are intentionally designed to accommodate, and even promote large-scale movement. Applications for such structures include seismic design, hurricane design, blast resistant design, deployable structures, responsive environments, art installations, et cetera. This research has only scraped the surface of what is possible for the future marriage of design and engineering.

\section{References}

[1] Leach, Neil, Turnbull, David and Williams, Chris. Digital Tectonics. Chichester, West Sussex, U.K.; Hoboken, NJ : Wiley-Academy, 2004.

[2] Shea, Kristina. "Directed Randomness," Digital Tectonics. Chichester, West Sussex, U.K.; Hoboken, NJ: Wiley-Academy, 2004.

[3] Adams, Vince, and Askenazi, Abraham. Building Better Products with Finite Element Analysis. Santa Fe: OnWard, 1999.

[4] Sabonnadiere, Jean-Claude, and Coulomb, Jean-Louis. Finite Element Methods in CAD. New York: Springer-Verlag, 1987.

[5] Adams, Vince, and Askenazi, Abraham. Building Better Products with Finite Element Analysis. Santa Fe: OnWard, 1999.

[6] Working method described from Altair Engineering Hypermesh Training Workshop, June 2006, Troy, Michigan. 